\title{
Does task shifting yield cost savings and improve efficiency for health systems? A systematic review of evidence from low-income and middle-income countries
}

\author{
Gabriel Seidman ${ }^{*}$ a and Rifat Atun
}

\begin{abstract}
Background: Task shifting has become an increasingly popular way to increase access to health services, especially in low-resource settings. Research has demonstrated that task shifting, including the use of community health workers (CHWs) to deliver care, can improve population health. This systematic review investigates whether task shifting in low-income and middle-income countries (LMICs) results in efficiency improvements by achieving cost savings.

Methods: Using the PRISMA guidelines for systematic reviews, we searched PubMed, Embase, CINAHL, and the Health Economic Evaluation Database on March 22, 2016. We included any original peer-review articles that demonstrated cost impact of a task shifting program in an LMIC.

Results: We identified 794 articles, of which 34 were included in our study. We found that substantial evidence exists for achieving cost savings and efficiency improvements from task shifting activities related to tuberculosis and HIV/AIDS, and additional evidence exists for the potential to achieve cost savings from activities related to malaria, NCDs, NTDs, childhood illness, and other disease areas, especially at the primary health care and community levels.

Conclusions: Task shifting presents a viable option for health system cost savings in LMICs. Going forward, program planners should carefully consider whether task shifting can improve population health and health systems efficiency in their countries, and researchers should investigate whether task shifting can also achieve cost savings for activities related to emerging global health priorities and health systems strengthening activities such as supply chain management or monitoring and evaluation.
\end{abstract}

Keywords: Task shifting, Community health workers, Health systems, Efficiency, Cost-effectiveness, Systematic review

\section{Background}

Efficient and effective health systems are critical for managing healthcare costs, addressing rising burden of disease, and providing sustainably universal health coverage. The efficiency of health spending has major implications for the health of the population. In low-income and middleincome countries (LMICs) of Africa, Asia, and the Middle East, increasing the efficiency of health spending could increase health-adjusted life expectancy by $1-2$ years [1].

\footnotetext{
*Correspondence: Gabriel.seidman@gmail.com

Department of Global Health and Population, Harvard T. H. Chan School of Public Health, 677 Huntington Avenue, Boston, MA 02115, United States of America
}

(c) The Author(s). 2017 Open Access This article is distributed under the terms of the Creative Commons Attribution 4.0 International License (http://creativecommons.org/licenses/by/4.0/), which permits unrestricted use, distribution, and reproduction in any medium, provided you give appropriate credit to the original author(s) and the source, provide a link to the Creative Commons license, and indicate if changes were made. The Creative Commons Public Domain Dedication waiver (http://creativecommons.org/publicdomain/zero/1.0/) applies to the data made available in this article, unless otherwise stated. cant portion of health expenditures; in LMICs, spending on salaried health workers makes up $28.7-33.2 \%$ of total health expenditure [2]. Improving the efficiency of spending on HRH can improve the efficiency of health systems, which can free up financial and other resources and ultimately improve health coverage [3].

According to the World Health Organization (WHO), task shifting "presents a viable solution for improving health care coverage by making more efficient use of the human resources already available and by quickly increasing capacity while training and retention programs are expanded" [4]. Task shifting can produce 
equivalent or superior outcomes for many diseases and health interventions including non-communicable diseases [5], HIV/AIDS [6, 7], contraceptive distribution [8], and others $[5,9]$.

Given the high spend on $\mathrm{HRH}$, the evidence for task shifting as a way to improve population health, and the prominence of task shifting on the global policy agenda, policymakers should understand the cost and efficiency implications of this approach to health systems strengthening (HSS). Therefore, our systematic review aims to answer the following question: Does task shifting result in cost savings and efficiency improvements for health systems or patients in LMICs?

To our knowledge, only one literature review has addressed a similar question so far [10]. That review found that community health workers (CHWs) are cost-effective for treating $\mathrm{TB}$ and select other disease areas, such as reproductive, maternal, newborn, and child health (RMNCH). Our review builds on the important initial review conducted by Vaughan et al. in three ways. First, our search strategy takes a broader scope in that it reviews other forms of task shifting besides the use of CHWs (e.g., shifting the work of physicians to nurses or the work of nurses to pharmacy technicians), which may contribute to HSS.

Second, our review looks at evidence for efficiency improvements achieved by shifting tasks from one cadre of workers to another, rather than whether an intervention using a specific type of health worker meets a cost-effectiveness threshold. Although costeffectiveness thresholds (e.g., cost/unit of health improvement above or below a pre-defined benchmark) are an important criterion for prioritizing interventions, cost-effectiveness as measured by an actual reduction in costs without a reduction in programmatic quality is particularly salient for policymakers trying to improve the efficiency of the health system. Therefore, we review whether studies found changes in cost per input/ process, output, or outcome as a result of task shifting. Whereas cost savings on inputs/processes are very likely since the wage for a lower-skilled worker will almost always be lower than that of a higher-skilled worker, cost savings on outputs and outcomes are not as guaranteed since lower-skilled workers might operate less efficiently. A reduction in cost per output or outcome can be interpreted as an improvement in efficiency and therefore a true savings to the health system (with changes in cost per outcome as the stronger indicator), but a reduction in cost per input/process can only be interpreted as an efficiency improvement if it is accompanied by the documentation of no change (or an improvement) in clinical or programmatic quality.

Third, following from the previous point, our review also captures and reports evidence of changes in programmatic or clinical quality as a result of task shifting for each included reference, which Vaughan et al. do not systematically report. Reporting programmatic quality outcomes is important for determining whether a reduction in costs actually indicates an improvement in health systems efficiency.

\section{Methods}

This systematic review follows the criteria and methodology described in the PRISMA guidelines on systematic reviews [11].

\section{Search process and criteria}

This search relied on an internal protocol developed by both authors, with the support of a Harvard University librarian specializing in systematic reviews. The protocol was not registered externally. We searched PubMed, Embase, CINAHL, and the Health Economic Evaluation Database. The main search that was conducted on March 22, 2016, was as follows (for PubMed), with an additional search term for LMICs, and any publication from before that data was eligible for our review:

(task shift*[tiab] OR balance of care[tiab] OR nonphysician clinician*[tiab] OR nonphysician clinician* [tiab] OR task sharing[tiab] OR community care giver* [tiab] OR community healthcare provider*[tiab] OR cadres[tiab] OR “Community Health Workers"[Mesh])

\section{AND}

("Cost Savings"[mesh] OR "Cost Benefit Analysis" [mesh] OR "Efficiency"[mesh] OR cost[tiab] OR costs [tiab] OR efficienc*[tiab] OR economies of scale[tiab] OR economies of scope[tiab] OR productivity[tiab] OR absenteeism[tiab] OR “Absenteeism”[Mesh])

We also conducted several additional searches based on a review of citation lists from relevant publications, and based on recommendations from public health researchers.

\section{Study selection and eligibility criteria}

After conducting our search, all titles were reviewed for relevance. After excluding irrelevant titles, we read all abstracts and, when appropriate, full articles to determine the relevance of the article for our research question. In order to be included in the study, the publication had to meet the following criteria:

- Report on an effort, such as a program or policy intervention, involving task shifting of a clinical activity or health systems-related activity

- Report a comparison of program costs from the task shifted model for conducting the activity or service to a comparable activity in a model that does not involve task shifting. 
- Report results from an actual intervention, rather than a computer model or simulation

- Report results from a low-income or middle-income country

- Be original research about an intervention published in a peer-reviewed format (as opposed to an editorial, literature review, opinion piece, interview, etc.)

- Have a complete article available (as opposed to just an abstract)

- Be published in English

\section{Data collection process}

In order to extract data for this review, we piloted an Excel-based data collection tool that was used to capture results from a preliminary search, the results of which were presented at the Harvard Ministerial Leadership Program in the summer of 2016. Based on our experience with this initial process, we modified the tool accordingly and finalized a tool which collected the following information: author, year, title, publication, abstract, country, continent, description of the intervention, main indicator, result on relevant indicator, and data on programmatic quality changes resulting from the intervention. Studies were not excluded if they did not have relevant quality comparisons. Results which did not provide evidence of cost changes, such as baseline costing studies, were excluded. GS conducted a first review of all references in the search, and the list was reviewed by RA and other public health researchers in order to identify missing references or references which had been improperly included.

We also retrospectively categorized the included references based on whether the main indicator documented changes in cost per input/process, output, or outcome, using the following definitions: [12].

- Inputs/processes: resources required to conduct an activity, or a discrete activity such as a patient visit with a clinician

- Outputs: direct products of program activities, such as number of individuals treated

- Outcomes: changes in health status as a result of the program, such as number of patients cured or number of deaths averted

\section{Risk of bias}

As with any systematic review, the references and data sources for this review contain the possibility for bias. At the level of individual references, authors are more likely to report cost data if their program resulted in cost savings, especially if costing/cost-effectiveness was not the primary purpose of the study.

Across all studies, there is also a risk of publication bias and selective reporting within studies, especially if authors more frequently chose to report positive outcomes (such as cost savings). Of course, the decision to implement task shifting in a given context would require extensive analysis of that particular intervention's potential impact, and we caution researchers and policymakers not to interpret the findings from this review as indicative of the results that they can expect to achieve.

\section{Results \\ Study selection}

We reviewed 791 articles and identified 34 references which analyzed the cost implications of task shifting in LMICs-22 in sub-Saharan Africa, eight in Asia and four in Central or South America. See Fig. 1 for the study selection for inclusion in this systematic review. Of the 32 studies included in the review by Vaughan et al., we excluded 17 and included 15, which means that our review also included an additional 19 studies not included in Vaughan et al. Of the 17 references included by Vaughan et al. that we excluded, 12 were excluded because they did not provide comparison of costs between the task shifted model and another model of care [13-24], three reported results from modeling of hypothetical programs rather than actual interventions [25-27], one reference did not have a full article available [28], and one reference reported the same data from the same program as another reference already included in our review [29].

Of the 34 studies included in our review, 30 found evidence of a reduction in health costs either to the health system or the patient, and four had a mixed impact, an increase in costs, or no changes in costs [30-33]. Almost all the studies focused on the effects of shifting clinical or public health tasks related to a specific disease or disease area, while one study focused on task shifting a HSS activity (mapping of village geographic coordinates) [34]. Only two studies examined task shifting within a hospital, whereas all others examined task shifting from the hospital to the primary health care (PHC) or community levels, or task shifting within the $\mathrm{PHC} /$ community level.

Of the 30 studies that found evidence of cost savings, 10 reported a cost savings per outcome, 13 reported a cost savings per output, and 3 reported a cost savings per input/process coupled with a corresponding maintenance or improvement in programmatic quality. Although cost savings on inputs/processes do not indicate efficiency improvements as strongly as savings on outputs or outcomes, the combined body of evidence from these 26 studies suggests that task shifting yields cost savings that result in efficiency improvements to the health system, especially at the PHC and community levels. The four citations which reported cost savings on an input/process and which did not report changes in clinical or programmatic quality all reported on tasks related to different disease areas/HSS activities. 


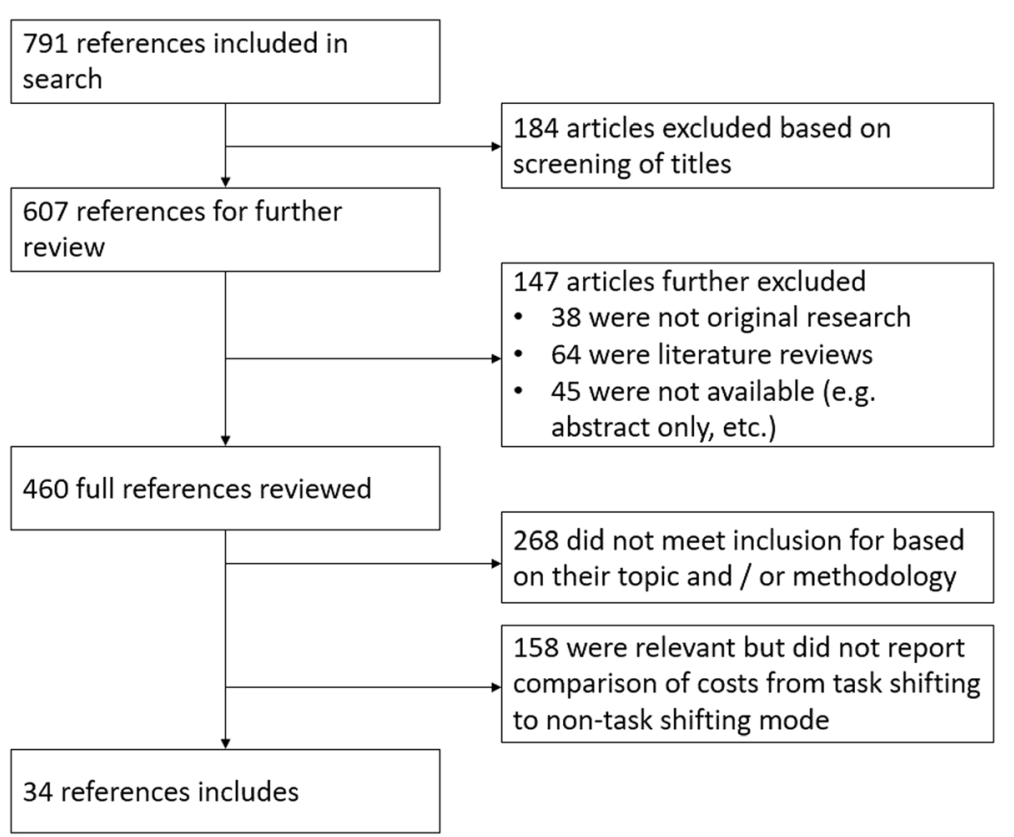

Fig. 1 Study selection for inclusion in systematic review

The full list of references meeting inclusion criteria can be found in Table 1 .

\section{Tuberculosis}

Nine studies demonstrated cost savings with task shifting for identification, diagnosis, and treatment of tuberculosis. Strategies for reducing costs included task shifting treatment supervision to health workers in the community [35-41], to home guardians or close relatives [42], laypersons [43], and in one case entrusting patients to take medicine without direct supervision [13]. Programmatic and clinical indicators, such as treatment success rate, treatment completion rate, and case finding rate, also indicate that task shifting programs maintained programmatic quality comparable or superior to traditional models of care.

\section{HIV/AIDS}

Studies in this review revealed cost savings from task shifting prevention and care for a high-risk group (men who have sex with men (MSM)) to community-based organizations [44], and dispensing of ART from pharmacists to Indirectly Supervised Pharmacist Assistants (ISPA), adherence clubs, or other pharmacy-only refill programs [45-48]. Programmatic indicators, such as patient retention, viral load, and mortality also indicate that these programs maintained high quality of care. These findings indicate that the dispensation of ARTs, especially to clinically stable patients who are very familiar with the routine of taking these drugs, is suitable for task shifting in low-resource (and possibly other) settings.
One study examining task shifting of ART dispensation to clinics found both an increase of costs in one state and a decrease in another state [31], and one study examining the task shifting initiation and management of ART treatment found no statistically significant differences in costs [30].

\section{Malaria}

Our review identified five articles that identified cost savings related to task shifting for malaria-related programs: CHW management of malaria $[49,50]$, village health worker (VHW) administration of IPT [51], community-based surveillance and treatment of malaria [52], and community-based surveillance and trapping of mosquitoes for vector control [53]. Indicators of program and clinical quality, such as administration of appropriate treatment, treatment completion rate, and average time from examination to initiation of treatment, indicate that the programs also maintained or improved programmatic quality. One study found a minor (9\%) increase in the cost of administration of IPT during pregnancy when shifting to a community-based model. Although the evidence is less robust than that for TB or HIV/AIDS, these findings suggest that many malaria-related tasks can achieve cost savings from task shifting.

\section{Other disease areas and activities}

Our review identified 11 additional studies which provided evidence of cost savings from task shifting for activities related to other diseases or health systems strengthening. These activities included controlling 


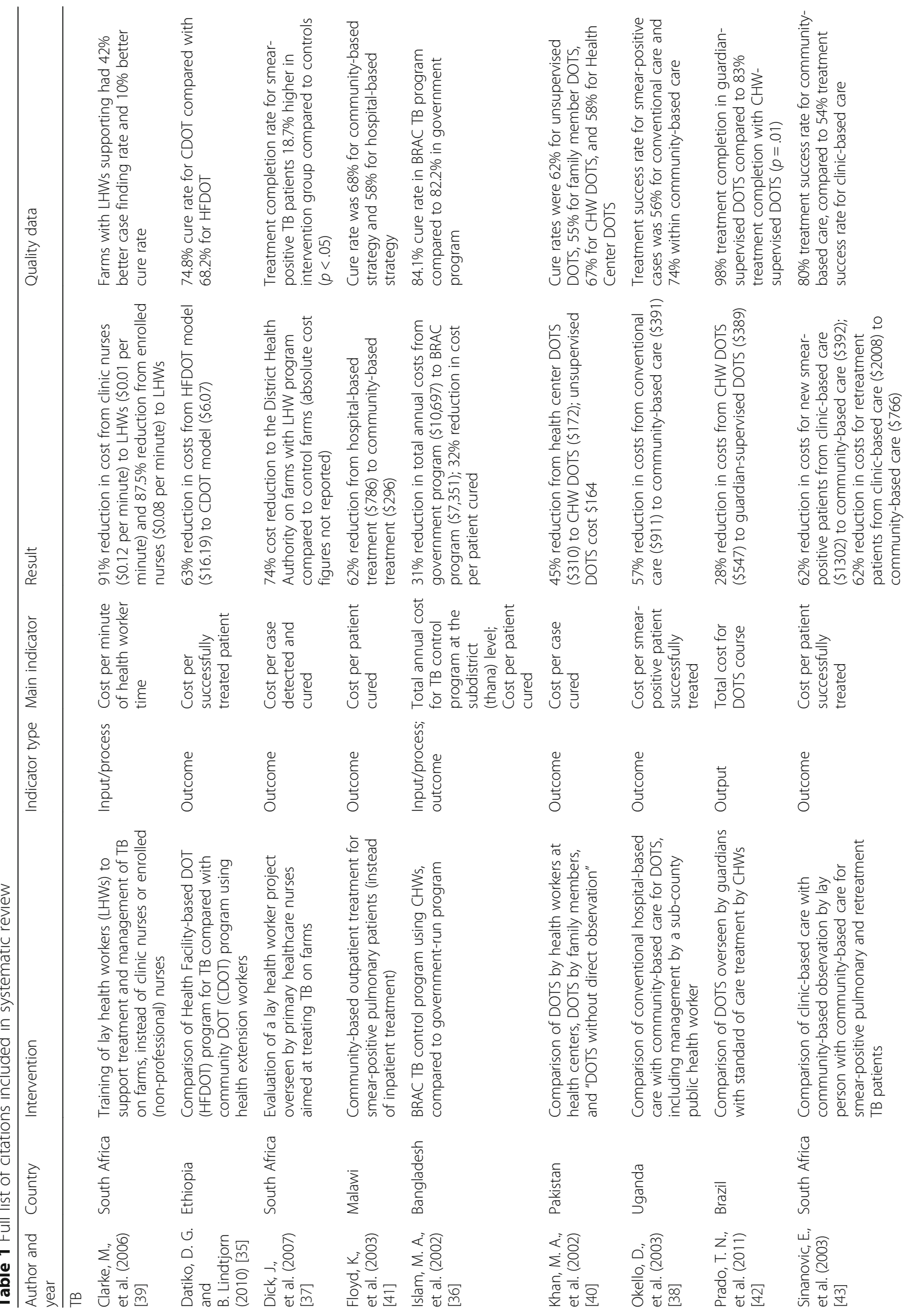




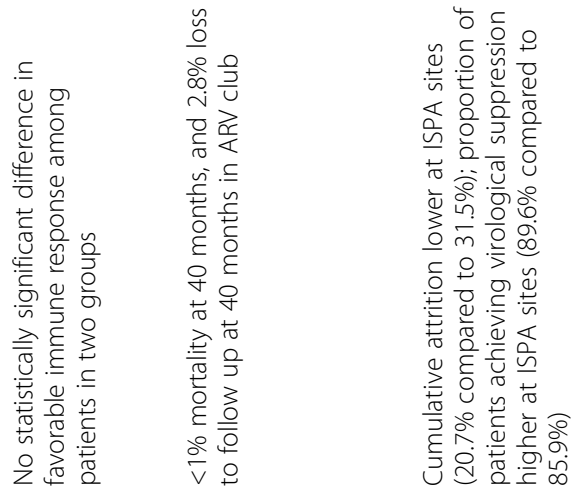

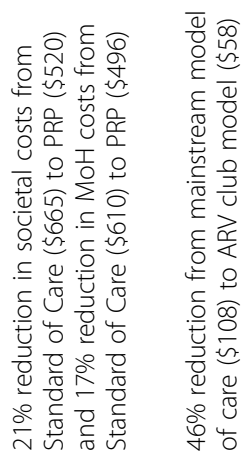

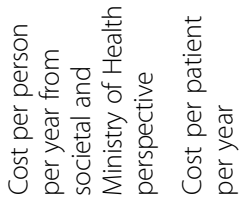

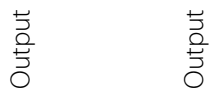

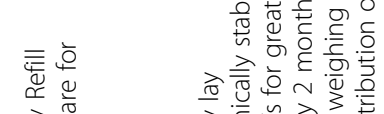

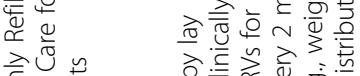

인.

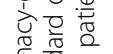

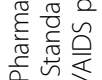

专

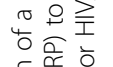

记

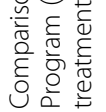

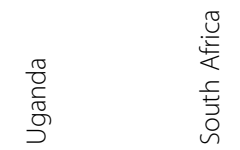

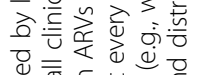

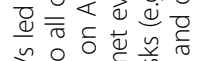

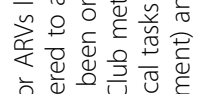

克离过

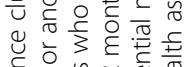

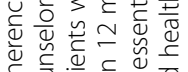

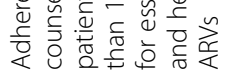

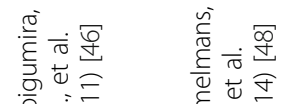

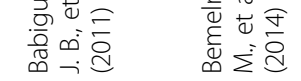

娄

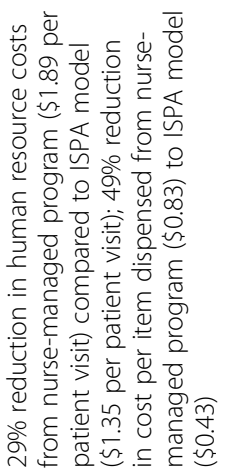

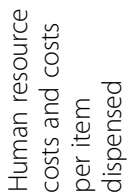

苍

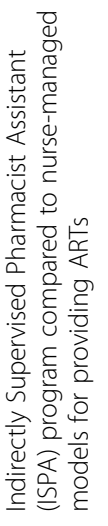

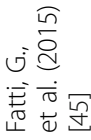

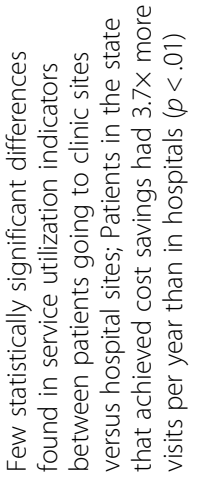

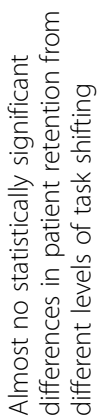

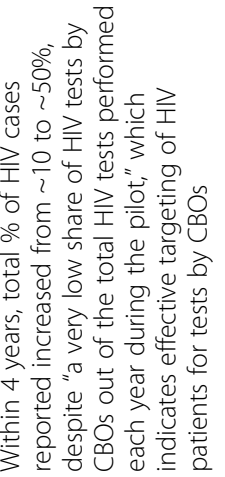

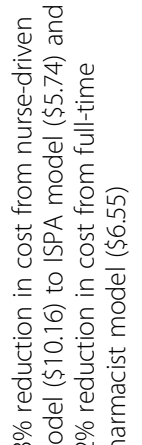

$\stackrel{\circ}{m} \subseteq \stackrel{\sharp}{0} \sum^{\tilde{n}}$

aे

苋管这.

过苋产离

至范完

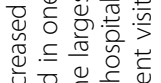

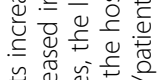

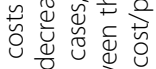

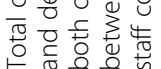

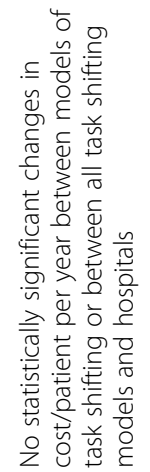

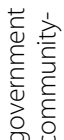

हก โ

을

守 声 $\tilde{c}$

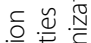

苋芼

م.

主

ष्ष

苍告

艺岂

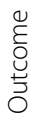

蒙

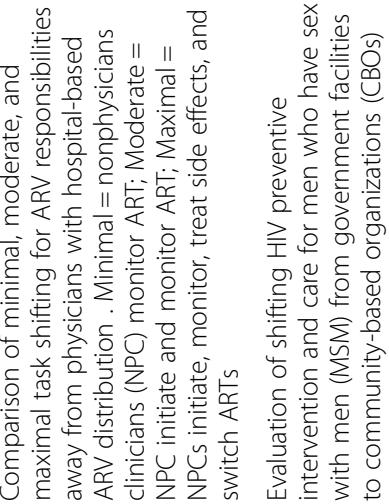

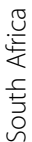

$\frac{\frac{.0}{2}}{\frac{0}{2}}$

$\frac{.0 \frac{0}{0}}{\frac{0}{\frac{0}{}}}$

胥

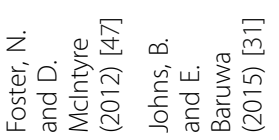

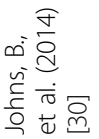

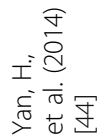




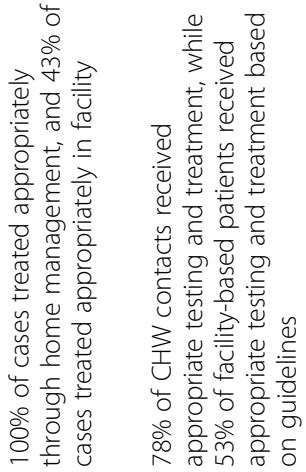

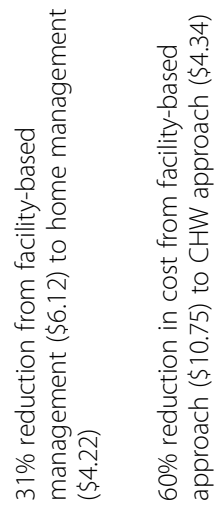

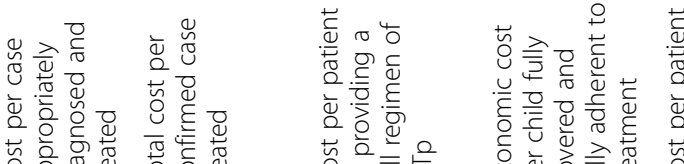
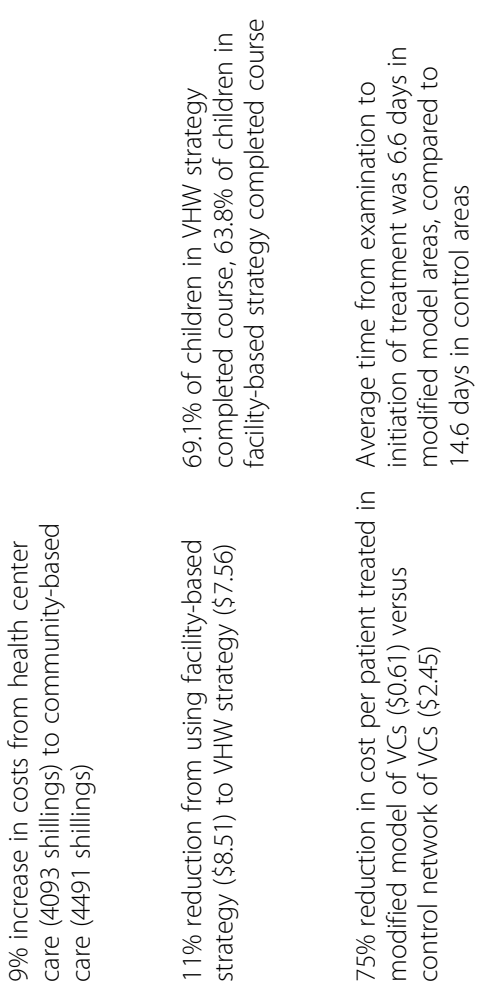

政

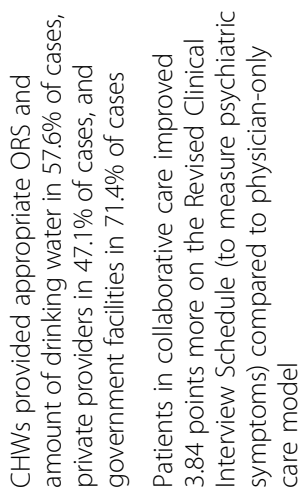

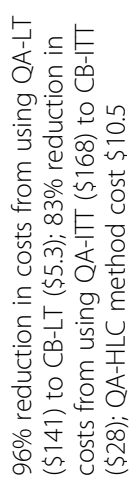

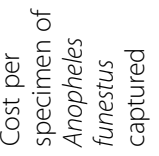

喜

$\frac{5}{2}$
$\frac{2}{5}$
0

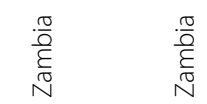

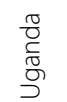

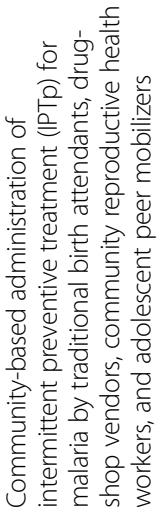

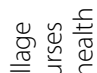

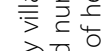

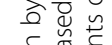

음 울

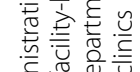

हो

金主离 离

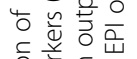

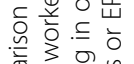

品

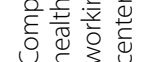

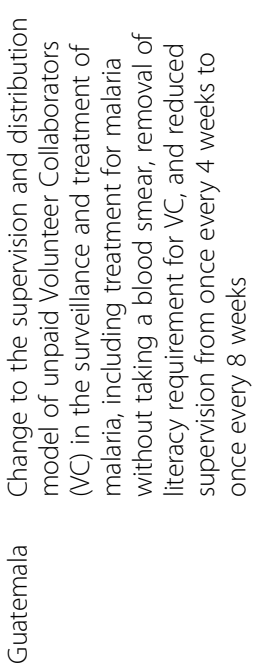

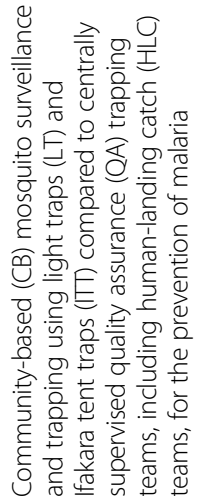

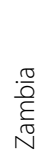

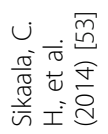

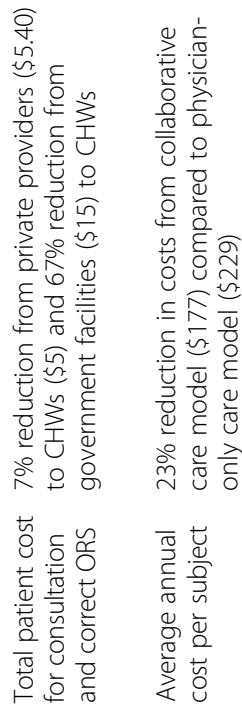

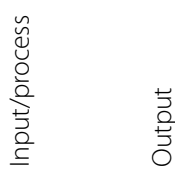
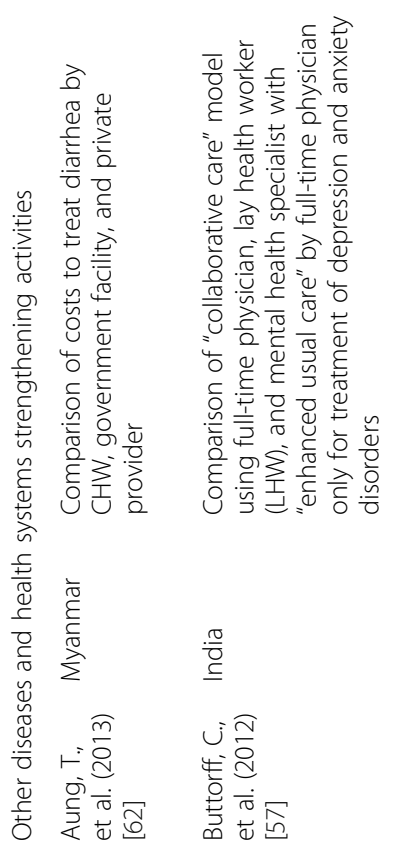

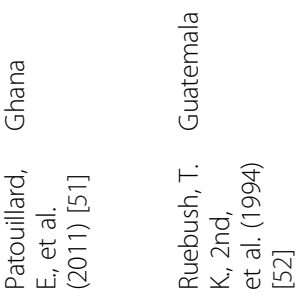




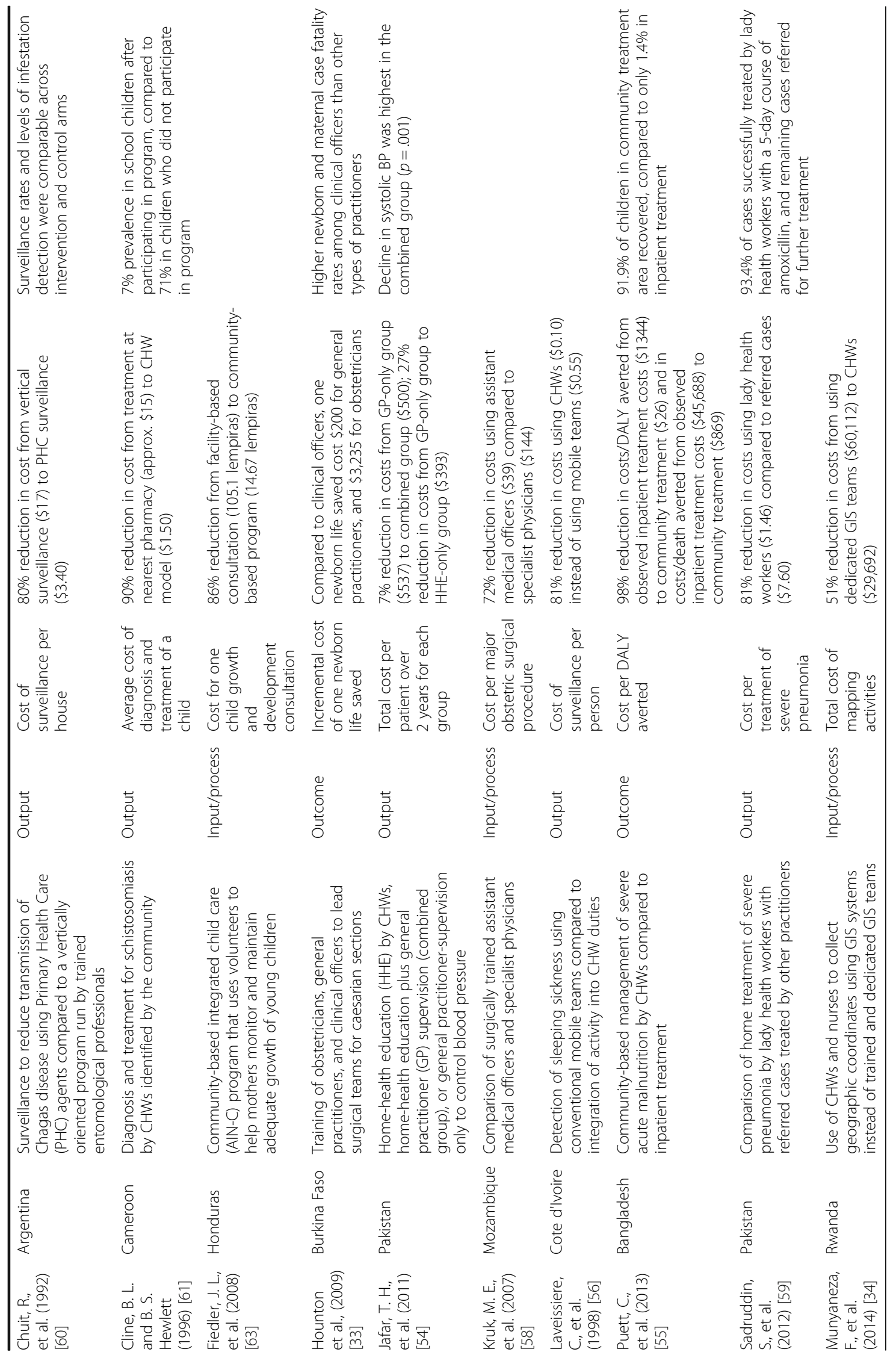


blood pressure through a combination of general practitioner and CHW activities [54], community-based management of severe acute malnutrition [55], integration of the detection of sleeping sickness intro routine CHW activities [56], treatment for mental health problems by a "collaborative care" team that included a lay health worker and mental health specialist [57], administration of major obstetric procedures by assistant medical officers instead of physicians [58], homebased treatment of severe pneumonia by lady health workers [59], integration of surveillance to reduce transmission of Chagas disease by Primary Health Care agents instead of specially trained professionals [60], diagnosis and treatment of schistosomiasis by $\mathrm{CHWs}$ [61], treatment of diarrhea by CHWs [62], communitybased integrated child care using volunteers to monitor and maintain growth [63], and geo-mapping activities by CHWs and nurses instead of dedicated GIS teams [34].

\section{Discussion}

This review aimed to identify whether task shifting can result in cost savings and efficiency improvements to health systems. Our results indicate that task shifting is a promising approach to achieving cost savings and improving efficiency in LMICs, and our results build on previous work which concluded that task shifting can be an effective way to improve population health. These findings have significant policy implications, discussed below, as well as important limitations.

\section{Task shifting can help achieve cost savings and} improve efficiency for activities related to top global health priorities, emerging global health issues, and neglected tropical diseases, but the evidence base is mostly limited to PHC and community-based care The most robust body of evidence found in this study is for achieving cost savings from task shifting activities related to TB and HIV/AIDS. Given the high burden of these diseases in LMICs and the longitudinal nature of preventing, treating, and managing these diseases, interventions that can reduce both their economic and health burdens simultaneously are particularly important for the future of global health. Each year there are 1.5 million new cases of tuberculosis, mostly in LMICs, and the global burden of TB amounts to approximately $\$ 12$ billion annually $[64,65]$. As of 2015, 36.7 million people were living with HIV, and meeting UNAIDS targets will require nearly $\$ 20$ billion annually $[66,67]$. TB treatment using DOTS is a relatively routine activity that occurs over many months and can take place in the community (when the infection is not drug-resistant).
Dispensation of ART to clinically stable patients who know and follow their drug regimens is also a relatively routine process. Therefore, these activities are well-suited for task shifting, and health systems can likely improve their efficiency by undertaking such efforts.

Outside of TB, HIV/AIDS, and malaria, the evidence for cost savings from task shifting was spread across many disease areas, making it difficult to conclude that task shifting activities for a specific disease could result in cost savings. Nonetheless, the fact that programs achieved cost savings from such a diverse set of diseases and across multiple geographies indicates that policymakers and program planners should consider task shifting as one of many potential approaches to improve efficiency in their health systems. The evidence for cost savings came from disease areas such as childhood illnesses, non-communicable diseases (which are receiving increased priority at the global level due to the Sustainable Development Goals), and neglected tropical diseases (NTDs). Almost all studies identified shifted tasks to or within the context primary health care (PHC) or community-based care. Although several citations identified cost savings by shifting tasks from hospitals to PHC or community care, only one citation found cost savings by shifting tasks within the hospital setting [58]. One additional study within the hospital setting found that shifting surgical care from physicians to clinical officers did not yield cost savings, but it did not analyze the cost-effectiveness of shifting surgical tasks from surgeons to other physicians [33]. While the body of evidence in this review suggests that task shifting can improve efficiency across multiple disease at the PHC and community levels, more research is needed on the effects of task shifting within secondary, tertiary, and highly specialized care.

2. Models of task shifting involve more than transferring clinical care to $\mathrm{CHWs}$

CHWs play a key role in reducing costs and increasing access to care in the health system. Nonetheless, this research shows that many models of task shifting exist outside of a simple transfer of clinical care to a CHW. Of course, many types of associate health professionals exist, such as pharmacy technicians, lay counsellors, and medical assistants, and the references included in this study reflect this diversity of health professions [68]. In particular, the use of different models for dispensing ART to HIV-positive patients was documented in multiple studies. In addition, several studies used models where CHWs or other 
lower-skilled workers collaborated with clinicians in order to provide a new model of care for the patient $[54,57]$.

Interestingly, only two studies identified cost savings from task shifting non-clinical activities: geo-mapping by CHWs and community-based mosquito trapping and surveillance. Given the importance that many non-clinical health systems functions have on improving population health (e.g., supply chain, monitoring and evaluation), research and program planners should consider the potential that task shifting could have for other health systems-related activities. For example, it is possible that lower-skilled professionals could perform routine tasks related to monitoring the supply chain or tracking patient data without compromising the quality of the activity.

3. The design and benefits of task shifting interventions will vary based on the context

Policymakers and program planners must recognize that task shifting is not a panacea for improving health and efficiency, but rather one of many tools to use in order to improve the efficiency of the health system. This review identified a range of task shifting models which resulted in different types of cost savings. Of course, without proper design, task shifting may actually increase system costs or reduce efficiency, such as by worsening overall population health due to poor clinical quality or increasing the number of staff in the health care system without changing care-seeking patterns among patients. Interestingly, one study found that the same model of task shifting resulted in both cost increases and cost decreases in two different regions of the same country [31]. Further, task shifting can also result in task overload for health workers, which could also reduce productivity and worsen health population health outcomes [69].

The breadth of task shifting models covered in this review is consistent with other findings from the literature which also indicate the need to adapt task shifting models to local contexts and health systems. For example, one systematic review notes a number of factors which can impact the success of lay health worker programs, including acceptability of the model to patients, implementation challenges such as problems with training, and health systems bottlenecks such as challenges with payment [70]. Another systematic review specifically identified strong management of $\mathrm{CHW}$ programs as the most important factor in their scale-up [71]. This body of evidence therefore suggests that designing appropriate task shifting models requires a thorough investigation of the local context, disease burden, and program goals.

\section{Limitations of the evidence, risks, and future directions for research}

There are several limitations to the research and its findings. First, this study includes citations that measure changes in cost and efficiency very differently. Of course, looking strictly at cost-effectiveness thresholds, rather than cost savings and programmatic indicators as a proxy for cost-effectiveness, would have helped to standardize these findings to make them more comparable. However, limiting our analysis to cost-effectiveness thresholds would also have negatively altered the evidence base in our review by (1) eliminating studies which demonstrated savings but did not have a formal cost-effectiveness analysis and (2) including studies that may have achieved some level of cost-effectiveness but which did not actually achieve savings (i.e., those in which an intervention by a specific cadre of health worker met a costeffectiveness threshold). By researching the impact of task shifting on costs to the health system as a proxy measure for efficiency improvements, we have focused on a key aspect of decision-making directly relevant to policymakers.

Second, unlike systematic reviews looking at health outcomes from highly specified clinical protocols, this review cannot predict the implications of a new task shifting program. Numerous factors in a given context will affect the outcomes of task shifting, including the burden of disease, the existing human resources for health, previous task shifting efforts, the social determinants of health, and the political economy of health. We caution that researchers and policymakers should not treat this review as a guarantee that future task shifting efforts will result in cost savings; rather, they should see this review as providing compelling evidence that task shifting can achieve cost savings if there is a need for such an intervention, and it is implemented appropriately.

Third, our search only identified two citations suitable for inclusion that examined task shifting within a hospital setting. Our search did not exclude programs that delivered services at a specific level, and the search included other citations focused on hospitals or specialty care that failed to meet inclusion criteria for other reasons (see select citations for examples [72-75]). This result suggests that the absence of evidence for task shifting within hospitals is likely due to the limited research on this topic to date. Nonetheless, LMICs have implemented programs to task shift hospital-based care, such as surgical services [76, 77]. Future research should examine models of task shifting within hospitals and their impacts on health outcomes, costs, and other relevant indicators.

Finally, as already discussed, the methodology of this review is limited by biases in reporting and publication of individual references. 
Going forward, we feel that researchers, program planners, and policymakers should continue to collaborate to understand both the financial and health impacts of task shifting. Many new task shifting efforts are underway globally, and ensuring that all these programs report on cost-effectiveness thresholds and changes in costs to the system will increase the evidence base surrounding this important topic. In particular, more programmatic research is needed to confirm the preliminary findings that task shifting for activities related to NCDs, NTDs, and health systems strengthening can result in cost savings, and to understand the role that task shifting can play in hospital and specialty settings. At the same time, researchers should also carefully examine the risk of task overload from task shifting and design ways to prevent and mitigate this risk.

\section{Conclusions}

This review examined the evidence for task shifting in improving health systems efficiency in LMICs. The evidence indicates that task shifting for activities across a broad range of diseases, including TB, HIV/AIDS, malaria, childhood illness, NCDs, and NTDs, can result in cost savings without compromising clinical or programmatic quality. This review also revealed that countries have used different approaches to introduce task shifting for management of different conditions and that task shifting takes on many forms besides simply transferring clinical activities to CHWs. Going forward, researchers, program planners, and policymakers should carefully examine their local context in order to determine whether task shifting can improve health systems efficiency while also maintaining or improving population health.

\section{Abbreviations}

ART: Antiretroviral therapy; CHW: Community health worker; DOTS: Directly observed treatment—-short course; HSS: Health systems strengthening; ISPA: Indirectly supervised pharmacist assistant; LMIC: Low-income and middleincome countries; MSM: Men who have sex with men; NCD: Noncommunicable disease; NTD: Neglected tropical disease; PHC: Primary health care; RMNCH:

Reproductive, maternal, newborn, and child health; TB: Tuberculosis;

VHW: Village health worker; WHO: World Health Organization

\section{Acknowledgements}

We thank Michael Sinclair and Brian Dugan from the Harvard Ministerial Leadership Program for their support in preparation of this report. We thank Paul Bain at Harvard University for assistance with designing the search strategy.

\section{Funding}

An original draft of this paper was commissioned by the Harvard Ministerial Leadership Program, a joint initiative of the Harvard TH Chan School of Public Health, Harvard Kennedy School of Government, and the Harvard Graduate School of Education in collaboration with Big Win Philanthropy, and with the support of the Bill and Melinda Gates Foundation, Bloomberg Philanthropies, the GE Foundation, and the Rockefeller Foundation.

\section{Availability of data and materials}

Key information from original dataset included in Table 1 is in the manuscript. The original dataset is available from the corresponding author upon request.

\section{Authors' contributions}

GS and RA jointly conceived of the research question, concept, and methodology for this paper. GS developed the data collection tool, reviewed all articles, and drafted and revised the manuscript. RA provided revisions and additional references for review. Both authors read and approved the final manuscript.

\section{Authors' information}

GS is a DrPH candidate at Harvard T. H. Chan School of Public Health. RA is the director of the Global Health Systems Cluster at Harvard T. H. Chan School of Public Health.

\section{Competing interests \\ The authors declare that they have no competing interests.}

\section{Consent for publication}

Not applicable.

Ethics approval and consent to participate

Not applicable.

\section{Publisher's Note}

Springer Nature remains neutral with regard to jurisdictional claims in published maps and institutional affiliations.

Received: 15 November 2016 Accepted: 29 March 2017

Published online: 13 April 2017

\section{References}

1. Grigoli F, Kapsoli J. Waste not, want not: the efficiency of health expenditure in emerging and developing economies. IMF Working Papers. 2013;(187).

2. Hernandez-Peña P. Health worker remuneration in WHO Member States. Bull World Health Organ. 2013;91(11):808-15.

3. Chisholm D, Evans DB. Improving health system efficiency as a means of moving towards universal coverage, in World Health Report Background Paper. Geneva: World Health Organization; 2010.

4. World Health Organization. First Global Conference on Task Shifting. 2008 [cited 2016 March 15]; Available from: http://www.who.int/healthsystems/ task_shifting/en/.

5. Joshi R, Alim M, Kengne AP, Jan S, Maulik PK, Peiris D, Patel AA. Task shifting for non-communicable disease management in low and middle income countries—a systematic review. PLoS One. 2014;9(8):e103754.

6. Kredo T, Adeniyi FB, Bateganya M, Pienaar ED. Task shifting from doctors to non-doctors for initiation and maintenance of antiretroviral therapy. Cochrane Database Syst Rev. 2014:7:Cd007331.

7. Penazzato M, Davies MA, Apollo T, Negussie E, Ford N. Task shifting for the delivery of pediatric antiretroviral treatment: a systematic review. J Acquir Immune Defic Syndr. 2014;65(4):414-22.

8. Polus S, Lewin S, Glenton C, Lerberg PM, Rehfuess E, Gülmezoglu AM. Optimizing the delivery of contraceptives in low- and middle-income countries through task shifting: a systematic review of effectiveness and safety. Reprod Health. 2015;12:27.

9. Martínez-González NA, Tandjung R, Djalali S, Rosemann T. The impact of physician-nurse task shifting in primary care on the course of disease: a systematic review. Hum Resour Health. 2015;13:55.

10. Vaughan K. Costs and cost-effectiveness of community health workers: evidence from a literature review. Hum Resour Health. 2015;13(1):1.

11. PRISMA. PRISMA: Transparent Reporting of Systematic Reviews and Meta-Analyses. 2015 [cited 2016 September 6]; Available from: http://www.prisma-statement.org/.

12. W. K. Kellogg Foundation. Logic Model Development Guide. Michigan: Battle Creek; 2004.

13. Alam K, Khan JA, Walker DG. Impact of dropout of female volunteer community health workers: an exploration in Dhaka urban slums. BMC Health Serv Res. 2012;12:260.

14. Borghi J. Economic assessment of a women's group intervention to improve birth outcomes in rural Nepal. Lancet. 2005;366(9500):1882-4.

15. Bowser $\mathrm{D}$, et al. A cost-effectiveness analysis of community health workers in Mozambique. J Pri Care Commun Health. 2015;6(4). 
16. Chin-Quee D. Building on safety, feasibility, and acceptability: the impact and cost of community health worker provision of injectable contraception. Global Health Sci Pract. 2013;1(3):316-27.

17. Chola L. Cost of individual peer counselling for the promotion of exclusive breastfeeding in Uganda. Cost Eff Resour Alloc. 2011;9(1):1.

18. Onwujekwe O, Uzochukwu B, Ojukwu J, Dike N, Shu E. Feasibility of a community health worker strategy for providing near and appropriate treatment of malaria in southeast Nigeria: an analysis of activities, costs and outcomes. Acta Trop. 2007;101(2):95-105.

19. Prinja S, Jeet G, Verma R, Kumar D, Bahuguna P, Kaur M, Kumar R. Economic analysis of delivering primary health care services through community health workers in 3 North Indian states. PLoS One. 2014;9(3):e91781.

20. Prinja S, Mazumder S, Taneja S, Bahuguna P, Bhandari N, Mohan P, Hombergh $\mathrm{H}$, Kumar R. Cost of delivering child health care through community level health workers: how much extra does IMNCl program cost? J Trop Pediatr. 2013;59(6):489-95.

21. Sabin LL. Costs and cost-effectiveness of training traditional birth attendants to reduce neonatal mortality in the Lufwanyama Neonatal Survival study (LUNESP). PLoS One. 2012;7(4):e35560.

22. Conteh L. Cost effectiveness of seasonal intermittent preventive treatment using amodiaquine \& artesunate or sulphadoxine-pyrimethamine in Ghanaian children. PLoS One. 2010;5(8):e12223.

23. Mahmud N, Rodriguez J, Nesbit J. A text message-based intervention to bridge the healthcare communication gap in the rural developing world. Technol Health Care. 2010;18(2):137-44.

24. Nonvignon J, Chinbuah MA, Gyapong M, Abbey M, Awini E, Gyapong JO, Aikins M. Is home management of fevers a cost-effective way of reducing under-five mortality in Africa? The case of a rural Ghanaian District. Trop Med Int Health. 2012;17(8):951-7.

25. Gaziano TA, Bertram M, Tollman SM, Hofman KJ. Hypertension education and adherence in South Africa: a cost-effectiveness analysis of community health workers. BMC Public Health. 2014;14:240.

26. McCord GC, Liu A, Singh P. Deployment of community health workers across rural sub-Saharan Africa: financial considerations and operational assumptions. Bull World Health Organ. 2013;91(4):244-53b.

27. Sutherland T, Bishai DM. Cost-effectiveness of misoprostol and prenatal iron supplementation as maternal mortality interventions in home births in rural India. Int J Gynecol Obstet. 2009;104(3):189-93.

28. Tozan Y, Klein EY, Darley S, Panicker R, Laxminarayan R, Breman JG. Pre-referral rectal artesunate is cost-effective for treating severe childhood malaria. Am J Trop Med Hyg. 2009;81(5):305.

29. Fiedler JL. A cost analysis of the Honduras community-based integrated child care program. Health, Nutrition and Population (HNP) Discussion Paper. Washington: The World Bank; 2003.

30. Johns B. Assessing the costs and effects of antiretroviral therapy task shifting from physicians to other health professionals in Ethiopia. J Acquir Immune Defic Syndr. 2014;65(4):e140-7.

31. Johns, B. and E. Baruwa, The effects of decentralizing anti-retroviral services in Nigeria on costs and service utilization: two case studies. Health Policy Plan. 2015;31(2):182-191.

32. Mbonye A. Intermittent preventive treatment of malaria in pregnancy: the incremental cost-effectiveness of a new delivery system in Uganda. Trans R Soc Trop Med Hyg. 2008;102(7):685-93.

33. Hounton SH. A cost-effectiveness study of caesarean-section deliveries by clinical officers, general practitioners and obstetricians in Burkina Faso. Hum Resour Health. 2009;7(1):34.

34. Munyaneza F. Leveraging community health worker system to map a mountainous rural district in low resource setting: a low-cost approach to expand use of geographic information systems for public health. Int J Health Geogr. 2014;13:49.

35. Datiko DG, Lindtjorn B. Cost and cost-effectiveness of smear-positive tuberculosis treatment by Health Extension Workers in Southern Ethiopia: a community randomized trial. PLoS One. 2010;5(2):e9158.

36. Islam MA. Cost-effectiveness of community health workers in tuberculosis control in Bangladesh. Bull World Health Organ. 2002;80(6):445-50.

37. Dick J. Primary health care nurses implement and evaluate a community outreach approach to health care in the South African agricultural sector. Int Nurs Rev. 2007;54(4):383-90.

38. Okello D. Cost and cost-effectiveness of community-based care for tuberculosis patients in rural Uganda. Int J Tuberc Lung Dis. 2003;7(9s1):S72-9.
39. Clarke M, Dick J, Bogg L. Cost-effectiveness analysis of an alternative tuberculosis management strategy for permanent farm dwellers in South Africa amidst health service contraction. Scand J Public Health. 2006;34(1):83-91.

40. Khan MA. Costs and cost-effectiveness of different DOT strategies for the treatment of tuberculosis in Pakistan. Directly Observed Treatment. Health Policy Plan. 2002;17(2):178-86.

41. Floyd K. Cost and cost-effectiveness of increased community and primary care facility involvement in tuberculosis care in Lilongwe District, Malawi. Int J Tuberc Lung Dis. 2003;7(9):S29-37.

42. Prado TN. Cost-effectiveness of community health worker versus homebased guardians for directly observed treatment of tuberculosis in Vitoria, Espirito Santo State, Brazil. Cad Saude Publica. 2011;27(5):944-52.

43. Sinanovic E. Cost and cost-effectiveness of community-based care for tuberculosis in Cape Town, South Africa. Int J Tuberc Lung Dis. 2003;7(9s1):S56-62.

44. Yan $\mathrm{H}$. The increased effectiveness of HIV preventive intervention among men who have sex with men and of follow-up care for people living with HIV after 'task-shifting' to community-based organizations: a 'cash on service delivery' model in China. PLoS One. 2014;9(7):e103146.

45. Fatti G, Monteith L, Shaikh N, Kapp E, Foster N, Grimwood A., A Comparison of Two Task-Shifting Models of Pharmaceutical Care in Antiretroviral Treatment Programs in South Africa. J Acquir Immune Defic Syndr. 2015;71(4).

46. Babigumira JB. Cost effectiveness of a pharmacy-only refill program in a large urban HIV/AIDS clinic in Uganda. PLoS One. 2011;6(3):e18193.

47. Foster N, Mclntyre D. Economic evaluation of task-shifting approaches to the dispensing of anti-retroviral therapy. Hum Resour Health. 2012;10:32.

48. Bemelmans M. Community-supported models of care for people on HIV treatment in sub-Saharan Africa. Trop Med Int Health. 2014;19(8):968-77.

49. Chanda P. Relative costs and effectiveness of treating uncomplicated malaria in two rural districts in Zambia: Implications for nationwide scale-up of homebased management. Malar J. 2011;10:159.

50. Hamainza B, Moonga H, Sikaala CH, Kamuliwo M, Bennett A, Eisele TP, Miller J, Seyoum A, Killeen GF. Monitoring, characterization and control of chronic, symptomatic malaria infections in rural Zambia through monthly household visits by paid community health workers. Malar J. 2014;13:128.

51. Patouillard E. Coverage, adherence and costs of intermittent preventive treatment of malaria in children employing different delivery strategies in Jasikan. Ghana PloS one. 2011;6(11):e24871.

52. Ruebush 2nd TK. Community participation in malaria surveillance and treatment. III. An evaluation of modifications in the Volunteer Collaborator Network of Guatemala. Am J Trop Med Hyg. 1994;50(1):85-98.

53. Sikaala $\mathrm{CH}$. A cost-effective, community-based, mosquito-trapping scheme that captures spatial and temporal heterogeneities of malaria transmission in rural Zambia. Malar J. 2014;13:225.

54. Jafar TH. Cost-effectiveness of community-based strategies for blood pressure control in a low-income developing country: findings from a cluster-randomized, factorial-controlled trial. Circulation. 2011;124(15):1615-25.

55. Puett $C$. Cost-effectiveness of the community-based management of severe acute malnutrition by community health workers in southern Bangladesh. Health Policy Plan. 2013;28(4):386-99.

56. Laveissiere $\mathrm{C}$. Detecting sleeping sickness: comparative efficacy of mobile teams and community health workers. Bull World Health Organ. 1998;76(6):559-64.

57. Buttorff C. Economic evaluation of a task-shifting intervention for common mental disorders in India. Bull World Health Organ. 2012;90(11):813-21.

58. Kruk ME. Economic evaluation of surgically trained assistant medical officers in performing major obstetric surgery in Mozambique. Bjog. 2007;114(10):1253-60

59. Sadruddin S. Household costs for treatment of severe pneumonia in Pakistan. Am J Trop Med Hyg. 2012;87(5 Suppl):137-43.

60. Chuit R. Result of a first step toward community-based surveillance of transmission of Chagas' disease with appropriate technology in rural areas. Am J Trop Med Hyg. 1992;46(4):444-50.

61. Cline BL, Hewlett BS. Community-based approach to schistosomiasis control. Acta Trop. 1996;61(2):107-19.

62. Aung T. Incidence of pediatric diarrhea and public-private preferences for treatment in rural Myanmar: a randomized cluster survey. J Trop Pediatr. 2013;59(1):10-6.

63. Fiedler $\mathrm{J}$, Villalobos CA, De Mattos AC. An activity-based cost analysis of the Honduras community-based, integrated child care (AIN-C) programme. Health Policy Plan. 2008;23(6):408-27. 
64. World Health Organization. Tuberculosis: Fact Sheet No. 104. 2015 [cited 2016 March 14]; Available from: http://www.who.int/mediacentre/ factsheets/fs104/en/.

65. Kim, J.Y. The burden of tuberculosis: Economic burden (2). 2016 [cited 2016 September 15]; Available from: http://who.int/trade/distance_learning/gpgh/ gpgh3/en/index7.html.

66. UNAIDS. Fact Sheet 2016. 2016 [cited 2016 September 16]; Available from: http://www.unaids.org/en/resources/fact-sheet.

67. UNAIDS. Fast-Track Update on Investments Needed in the AIDS Response. 2016 [cited 2016 September 15]; Available from: http://www.unaids.org/ sites/default/files/media_asset/UNAIDS_Reference_FastTrack_Update_on_ investments_en.pdf.

68. World Health Organization, Classifying health workers: Mapping occupations to the international standard classification. Geneva: World Health Organization.

69. Jaskiewicz W, Tulenko K. Increasing community health worker productivity and effectiveness: a review of the influence of the work environment. Hum Resour Health. 2012;10(1):1.

70. Glenton C, et al. Barriers and facilitators to the implementation of lay health worker programmes to improve access to maternal and child health: qualitative evidence synthesis. Cochrane Database Syst Rev. 2013;10: Cd010414.

71. Pallas SW. Community health workers in low- and middle-income countries: what do we know about scaling up and sustainability? Am J Public Health. 2013;103(7):e74-82.

72. Bastawrous A, Giardini ME, Bolster NM, Peto T, Shah N, Livingstone IA, Weiss HA, Hu S, Rono H, Kuper H, Burton M. Clinical Validation of a Smartphone-Based Adapter for Optic Disc Imaging in Kenya. JAMA Ophthalmol. 2016;134(2).

73. Gupta B, Huckman RS, Khanna T. Task shifting in surgery: lessons from an Indian Heart Hospital. Healthc (Amst). 2015;3(4):245-50.

74. Boullé C, Kouanfack C, Laborde-Balen G, Carrieri MP, Dontsop M, Boyer S, Aghokeng AF, Spire B, Koulla-Shiro S, Delaporte E, Laurent C. Task shifting HIV care in rural district hospitals in Cameroon: evidence of comparable antiretroviral treatment-related outcomes between nurses and physicians in the Stratall ANRS/ESTHER trial. J Acquir Immune Defic Syndr. 2013;62(5):569-76.

75. Nash D, Azeez S, Vlahov D, Schori M. Evaluation of an intervention to increase screening colonoscopy in an urban public hospital setting. J Urban Health. 2006;83(2):231-43.

76. Mullan F, Frehywot S. Non-physician clinicians in 47 sub-Saharan African countries. Lancet. 2007;370(9605):2158-63.

77. Chu K. Surgical task shifting in sub-Saharan Africa. PLoS Med. 2009;6(5):e1000078.

\section{Submit your next manuscript to BioMed Central and we will help you at every step:}

- We accept pre-submission inquiries

- Our selector tool helps you to find the most relevant journal

- We provide round the clock customer support

- Convenient online submission

- Thorough peer review

- Inclusion in PubMed and all major indexing services

- Maximum visibility for your research

Submit your manuscript at www.biomedcentral.com/submit

C) Biomed Central 